UDC 517.98

\author{
C-C. Chen, S. M. Tabatabaie
}

\title{
CHAOTIC AND HYPERCYCLIC OPERATORS ON SOLID BANACH FUNCTION SPACES
}

\begin{abstract}
In this paper, we study hypercyclicity on solid Banach function spaces, and give the characterization for weighted translation operators to be hypercyclic in terms of weight and aperiodic functions. Some sufficient and necessary conditions for these operators to be chaotic are obtained as well.
\end{abstract}

Key words: hypercyclic operator, chaotic operator, weighted translation operator, solid Banach function space, aperiodic function

2010 Mathematical Subject Classification: $47 A 16$

1. Introduction and Preliminaries. Hypercyclicity, as an active research topic in mathematics, arises from the invariant closed subset problem, which is one of the important and significant problems in analysis. Besides, it is related to some notions of topological dynamics, such as topological transitivity, topological mixing, linear chaos, and so on. Indeed, fruitful results and theories appeared during the last four decades. We refer to these two classic books [2], [10] as monographs on this topic.

Among the works in this direction, H. Salas in [14] gave a concrete example on $\ell^{p}(\mathbb{Z})$ by characterizing hypercyclic weighted shifts, which is very important to motivate researchers to demonstrate some deep results and construct examples in various cases. Inspired by H. Salas' work, the authors of [6], [7] gave some sufficient and necessary conditions for weighted translation operators to be hypercyclic on the Lebesgue space in context of homogeneous spaces and locally compact groups.

Recently, the focus was on studying hypercyclicity of operators on other special function spaces, such as Orlicz spaces; see [8], [9]. In this note, we consider hypercyclicity on more general spaces, namely, Banach function spaces, which are Banach spaces of measurable functions. Indeed, we characterize hypercyclic weighted translation operators on such spaces.

(c) Petrozavodsk State University, 2020 
Also, we give some sufficient and necessary conditions for these operators to be chaotic, and present some application in Morrey spaces.

First, we recall some preliminaries and definitions of Banach function spaces, and refer to [3] for a classic monograph. Let $X$ be a topological space. Denote the set of all Borel measurable complex-valued functions on $X$ by $\mathcal{M}_{0}(X)$.

Definition 1. Let $X$ be a topological space and $\mathcal{F}$ be a linear subspace of $\mathcal{M}_{0}(X)$. If $\mathcal{F}$ equipped with a given norm $\|\cdot\|_{\mathcal{F}}$ is a Banach space, we say that $\mathcal{F}$ is a Banach function space on $X$. A Banach function space $\left(\mathcal{F},\|\cdot\|_{\mathcal{F}}\right)$ on $X$ is called solid if for each $f \in \mathcal{F}$ and $g \in \mathcal{M}_{0}(X)$, the inequality $|g| \leqslant|f|$ implies $g \in \mathcal{F}$ and $\|g\|_{\mathcal{F}} \leqslant\|f\|_{\mathcal{F}}$.

Definition 2. Let $\mathcal{F}$ be a Banach function space on a topological space $X$, and $\alpha: X \rightarrow X$ be a Borel measurable bijection, whose inverse $\alpha^{-1}$ is also Borel measurable. We say that $\mathcal{F}$ is $\alpha$-invariant if $f \circ \alpha^{ \pm 1} \in \mathcal{F}$ and $\left\|f \circ \alpha^{ \pm 1}\right\|_{\mathcal{F}}=\|f\|_{\mathcal{F}}$ for each $f \in \mathcal{F}$.

Example 1. Let $G$ be a locally compact group, $a$ be a fixed element in $G$, and $\Phi$ be a Young function. Let $\left(L^{\Phi}(G),\|\cdot\|_{\Phi}\right)$ be an Orlicz space with respect to a left Haar measure on $G$. Define the function $\alpha_{a}: G \rightarrow G$ by

$$
\alpha_{a}(x):=a x, \quad(x \in G) .
$$

Then $L^{\Phi}(G)$ is an $\alpha_{a}$-invariant solid Banach function space on $G$.

In the following, we recall the definition of hypercyclicity.

Definition 3. Let $\mathcal{X}$ be a Banach space. A bounded linear operator $T: \mathcal{X} \rightarrow \mathcal{X}$ is called hypercyclic if there exists an element $x \in \mathcal{X}$, such that the orbit $\left\{x, T x, \ldots, T^{n} x, \ldots\right\}$ is dense in $\mathcal{X}$; here $T^{n}$ denotes the $n$-th iterate of $T$. In this case, $x$ is called a hypercyclic vector.

It is well-known (cf. [10]) that topological transitivity and hypercyclicity are equivalent on $\mathcal{X}$. An operator $T$ is said to be topologically transitive if for any pair of non-empty open sets $U, V$ in $\mathcal{X}$, there exists $n \in \mathbb{N}$, such that $T^{n}(U) \cap V \neq \varnothing$. Besides, it should be noted that a Banach space admits a hypercyclic operator if, and only if, it is separable and infinitedimensional [1], [4]. So, in this paper, we assume that Banach spaces are separable and infinite-dimensional. Next, we introduce our setting.

Definition 4. Let $X$ be a topological space and $\alpha: X \rightarrow X$ be a Borel measurable bijection, such that its inverse is also Borel measurable. Let $\mathcal{F}$ be an $\alpha$-invariant solid Banach function space on $X$ and let 
$w: X \rightarrow(0, \infty)$ be a bounded Borel measurable function (called a weight). Then, the corresponding (generated) weighted translation operator $T_{\alpha, w}$ on $\mathcal{F}$ is defined by

$$
T_{\alpha, w}: \mathcal{F} \rightarrow \mathcal{F}, \quad T_{\alpha, w} f:=w \cdot(f \circ \alpha)
$$

for all $f \in \mathcal{F}$.

Easily, one can see that $T_{\alpha, w}$ is well-defined, and by some calculation, for each $n \in \mathbb{N}$ and $f \in \mathcal{F}$, we have

$$
T_{\alpha, w}^{n} f=\left(\prod_{j=0}^{n-1} w \circ \alpha^{j}\right) \cdot\left(f \circ \alpha^{n}\right)
$$

where $\alpha^{n}$ means $n$-fold combination of $\alpha$. If $\frac{1}{w}$ is also bounded, then $T_{\alpha, w}$ is invertible and we denote its inverse by $S_{\alpha, w}:=T_{\alpha, w}^{-1}$.

In order to pursue our goal, one more concept is required.

Definition 5. Let $X$ be a topological space, and $\alpha: X \rightarrow X$ be a Borel measurable bijection, such that its inverse is also Borel measurable. Then $\alpha$ is called aperiodic if for each compact subset $K$ of $X$ there exists a constant $N>0$, such that for each $n \geqslant N, K \cap \alpha^{ \pm n}(K)=\varnothing$.

Example 2. Aperiodicity of elements of a locally compact group $G$ was defined originally in [11]. In fact, an element $a \in G$ is called compact in [11]) if the closed subgroup of $G$ generated by $a$ is compact, and any non-compact element of $G$ is called aperiodic in [7]. Let $G$ be a second countable locally compact group; fix an element $a \in G$. As in Example 1, define the function $\alpha_{a}: G \rightarrow G$ by $\alpha_{a}(x):=a x$ for all $x \in G$. Then, by the characterization given in [7, Lemma 2.1], $\alpha_{a}$ is an aperiodic function if, and only if, $a$ is an aperiodic element of $G$. We note (see [7]) that in many familiar non-discrete groups, including the additive group $\mathbb{R}^{d}$, the Heisenberg group and the affine group, all elements except the identity are aperiodic. Therefore, one can construct many aperiodic functions $\alpha$ on various groups.

2. Main Results. In this section, we will provide and demonstrate the main results. We denote by $\chi_{E}$ the characteristic function of $E$. To remind ourselves of all the required conditions and properties on the Banach function space $\mathcal{F}$, we collect them below.

Definition 6. Let $X$ be a topological space, $\mathcal{F}$ be a Banach function space on $X$, and $\alpha$ be a Borel measurable bijection from $X$ onto $X$, such 
that $\alpha^{-1}$ is also Borel measurable. Let $\mathcal{F}_{b c}$ be the set of all bounded compactly supported functions in $\mathcal{F}$. We say that $\mathcal{F}$ satisfies condition $\Omega_{\alpha}$ if

1) $\mathcal{F}$ is solid and $\alpha$-invariant;

2) for each compact set $E \subseteq X$, we have $\chi_{E} \in \mathcal{F}$;

3) $\mathcal{F}_{b c}$ is dense in $\mathcal{F}$.

Example 3. Let $G$ be a locally compact group, $a$ be a fixed element in $G$ and $\alpha_{a}$ be the mapping given in Example 1. Let $\Phi$ be a $\Delta_{2}$-regular Young function. Then the Orlicz space $L^{\Phi}(G)$ satisfies condition $\Omega_{\alpha_{a}}$. We recall that a Young function $\Phi$ is called $\Delta_{2}$-regular if there are some constants $c>0$ and $x_{0} \geqslant 0$, such that $\Phi(2 x) \leqslant c \Phi(x)$ for all $x \geqslant x_{0}$.

Now we are ready to give the main results of this paper.

Theorem 1. Let $X$ be a topological space and $\alpha$ be an aperiodic function on $X$. Let $w$ be a given weight on $X$, and $\frac{1}{w}$ be bounded. Let $\mathcal{F}$ be a Banach function space on $X$ satisfying condition $\Omega_{\alpha}$. Then the following properties are equivalent.

(i) $T_{\alpha, w}$ is hypercyclic on $\mathcal{F}$.

(ii) For each compact subset $K$ of $X$, there are a sequence of Borel subsets $\left(E_{k}\right)_{k=1}^{\infty}$ of $K$, and a strictly increasing sequence of natural numbers $\left(n_{k}\right)_{k=1}^{\infty}$, such that $\lim _{k \rightarrow \infty}\left\|\chi_{K \backslash E_{k}}\right\|_{\mathcal{F}}=0$ and

$$
\lim _{k \rightarrow \infty} \sup _{x \in E_{k}} \prod_{j=0}^{n_{k}-1}\left(\left(w \circ \alpha^{j}\right)(x)\right)^{-1}=\lim _{k \rightarrow \infty} \sup _{x \in E_{k}} \prod_{j=1}^{n_{k}}\left(w \circ \alpha^{-j}\right)(x)=0 .
$$

Proof. (i) $\Rightarrow$ (ii). Suppose that $T_{\alpha, w}$ is hypercyclic, and $K \subseteq X$ is compact. Then, by the condition (2) of Definition 6, we have $\chi_{K} \in \mathcal{F}$. Since $\alpha$ is an aperiodic function, there exists a constant $M>0$, such that for each $n \geqslant M$,

$$
K \cap \alpha^{ \pm n}(K)=\varnothing
$$

Since $T_{\alpha, w}$ is hypercyclic, the set of all hypercyclic vectors of $T_{\alpha, w}$ is dense in $\mathcal{F}$. So, for each $k \in \mathbb{N}$, there is a hypercyclic vector $f_{k}$ in $\mathcal{F}$, such that

$$
\left\|f_{k}-\chi_{K}\right\|_{\mathcal{F}} \leqslant \frac{1}{4^{k}}
$$

Due to the fact that $f_{k}$ is a hypercyclic vector, there is a natural number $n_{k}$, such that

$$
\left\|T_{\alpha, w}^{n_{k}} f_{k}-\chi_{K}\right\|_{\mathcal{F}}<\frac{1}{4^{k}}
$$


We can suppose that $n_{1}>M$, and the sequence $\left(n_{k}\right)_{k=1}^{\infty}$ is strictly increasing. Put $A_{k}:=\left\{x \in K:\left|f_{k}(x)-1\right| \geqslant \frac{1}{2^{k}}\right\}$. Then, for each $x \in K \backslash A_{k}$, we have

$$
1-\left|f_{k}(x)\right| \leqslant\left|f_{k}(x)-1\right|<\frac{1}{2^{k}},
$$

and so, $0<1-\frac{1}{2^{k}}<\left|f_{k}(x)\right|$. Thus,

$$
\left\|\chi_{A_{k}} f_{k}-\chi_{A_{k}}\right\|_{\mathcal{F}} \leqslant\left\|f_{k}-\chi_{K}\right\|_{\mathcal{F}} \leqslant \frac{1}{4^{k}}
$$

which is implied by the fact that $f_{k}-\chi_{K} \in \mathcal{F}$, the solidity of $\mathcal{F}$, and

$$
\left|\chi_{A_{k}} f_{k}-\chi_{A_{k}}\right|=\left|\chi_{A_{k}} f_{k}-\chi_{A_{k}} \chi_{K}\right|=\chi_{A_{k}}\left|f_{k}-\chi_{K}\right| \leqslant\left|f_{k}-\chi_{K}\right| .
$$

Moreover,

$$
\left|\frac{1}{2^{k}} \chi_{A_{k}}\right| \leqslant\left|\chi_{A_{k}} f_{k}-\chi_{A_{k}}\right|
$$

which entails

$$
\left\|\frac{1}{2^{k}} \chi_{A_{k}}\right\|_{\mathcal{F}} \leqslant\left\|\chi_{A_{k}} f_{k}-\chi_{A_{k}}\right\|_{\mathcal{F}}
$$

By (4) and (5), we have $\left\|\chi_{A_{k}}\right\|_{\mathcal{F}} \leqslant \frac{1}{2^{k}}$. Now, we put $B_{k}:=\{x \in X \backslash K$ : $\left.\left|f_{k}(x)\right| \geqslant \frac{1}{2^{k}}\right\}$. Then, for each $x \in X \backslash\left(K \cup B_{k}\right)$, we have $\left|f_{k}(x)\right|<\frac{1}{2^{k}}$. Also, since $B_{k} \cap K=\varnothing$, we have

$$
\begin{aligned}
\left|\chi_{B_{k}} f_{k}\right|=\left|\chi_{B_{k}} f_{k}-\chi_{B_{k} \cap K}\right|=\left|\chi_{B_{k}} \cdot\left(f_{k}-\chi_{K}\right)\right| & = \\
& =\chi_{B_{k}}\left|f_{k}-\chi_{K}\right| \leqslant\left|f_{k}-\chi_{K}\right|,
\end{aligned}
$$

which implies $\chi_{B} f_{k} \in \mathcal{F}$ by solidity of $\mathcal{F}$. Therefore,

$$
\left\|\chi_{B_{k}} f_{k}\right\|_{\mathcal{F}} \leqslant\left\|f_{k}-\chi_{K}\right\|_{\mathcal{F}} \leqslant \frac{1}{4^{k}}
$$

Besides, by the definition of $B_{k}$, we have $0 \leqslant \frac{1}{2^{k}} \chi_{B_{k}} \leqslant \chi_{B_{k}}\left|f_{k}\right|$. So,

$$
\left\|\frac{1}{2^{k}} \chi_{B_{k}}\right\|_{\mathcal{F}} \leqslant\left\|\chi_{B_{k}} f_{k}\right\|_{\mathcal{F}}
$$

Using (6) and (7), we have $\left\|\chi_{B_{k}}\right\|_{\mathcal{F}} \leqslant \frac{1}{2^{k}}$. On the other hand, for all $k \in \mathbb{N}$, 


$$
\begin{gathered}
\frac{1}{4^{k}}>\left\|T_{\alpha, w}^{n_{k}} f_{k}-\chi_{K}\right\|_{\mathcal{F}}=\left\|\left(\prod_{j=0}^{n_{k}-1} w \circ \alpha^{j}\right) \cdot\left(f_{k} \circ \alpha^{n_{k}}\right)-\chi_{K}\right\|_{\mathcal{F}}= \\
=\left\|\left(\prod_{j=0}^{n_{k}-1} w \circ \alpha^{j} \circ \alpha^{-n_{k}}\right) f_{k}-\left(\chi_{K} \circ \alpha^{-n_{k}}\right)\right\|_{\mathcal{F}}= \\
=\left\|\left(\prod_{j=1}^{n_{k}} w \circ \alpha^{-j}\right) f_{k}-\left(\chi_{K} \circ \alpha^{-n_{k}}\right)\right\|_{\mathcal{F}}
\end{gathered}
$$

For each $k \in \mathbb{N}$, put

$$
C_{k}:=\left\{x \in K:\left|\left(\prod_{j=0}^{n_{k}-1}\left(w \circ \alpha^{j}\right)(x)\right) \cdot\left(f_{k} \circ \alpha^{n_{k}}\right)(x)-1\right| \geqslant \frac{1}{2^{k}}\right\} .
$$

Then, by (3) and the solidity of $\mathcal{F}$, we have

$$
\begin{aligned}
& \frac{1}{4^{k}}>\left\|\left(\prod_{j=0}^{n_{k}-1} w \circ \alpha^{j}\right) \cdot\left(f_{k} \circ \alpha^{n_{k}}\right)-\chi_{K}\right\|_{\mathcal{F}} \geqslant \\
& \geqslant\left\|\chi_{C_{k}}\left(\prod_{j=0}^{n_{k}-1} w \circ \alpha^{j}\right) \cdot\left(f_{k} \circ \alpha^{n_{k}}\right)-\chi_{C_{k}}\right\|_{\mathcal{F}} \geqslant\left\|\frac{1}{2^{k}} \chi_{C_{k}}\right\|_{\mathcal{F}}=\frac{1}{2^{k}}\left\|\chi_{C_{k}}\right\|_{\mathcal{F}}
\end{aligned}
$$

which says $\left\|\chi_{C_{k}}\right\|_{\mathcal{F}}<\frac{1}{2^{k}}$. Next, set

$$
D_{k}:=\left\{x \in K:\left(\prod_{j=1}^{n_{k}}\left(w \circ \alpha^{-j}\right)(x)\right) \cdot\left|f_{k}(x)\right| \geqslant \frac{1}{2^{k}}\right\} .
$$

If $x \notin K$, then clearly, $\chi_{D_{k}}(x) \cdot \chi_{K}\left(\alpha^{-n_{k}}(x)\right)=0$. Let $x \in K$. By aperiodicity of $\alpha$, one has $\alpha^{-n_{k}}(K) \cap K=\varnothing$, and so $\alpha^{-n_{k}}(x) \notin K$. Hence $\chi_{D_{k}}(x) \cdot \chi_{K}\left(\alpha^{-n_{k}}(x)\right)=0$. Together with (3), this imply

$$
\begin{aligned}
\frac{1}{4^{k}}>\left\|\left(\prod_{j=1}^{n_{k}} w \circ \alpha^{-j}\right) f_{k}-\left(\chi_{K} \circ \alpha^{-n_{k}}\right)\right\|_{\mathcal{F}} & =\left\|\chi_{D_{k}}\left(\prod_{j=1}^{n_{k}} w \circ \alpha^{-j}\right) f_{k}\right\|_{\mathcal{F}} \geqslant \\
& \geqslant\left\|\frac{1}{2^{k}} \chi_{D_{k}}\right\|_{\mathcal{F}}=\frac{1}{2^{k}}\left\|\chi_{D_{k}}\right\|_{\mathcal{F}}
\end{aligned}
$$

which implies $\left\|\chi_{D_{k}}\right\|_{\mathcal{F}}<\frac{1}{2^{k}}$. 
Next, we show that for each $x \in K \backslash\left(C_{k} \cup \alpha^{-n_{k}}\left(B_{k}\right)\right)$,

$$
\left(\prod_{j=0}^{n_{k}-1} w \circ \alpha^{j}(x)\right)^{-1} \leqslant \frac{\left|f_{k} \circ \alpha^{n_{k}}(x)\right|}{1-\frac{1}{2^{k}}}<\frac{\frac{1}{2^{k}}}{1-\frac{1}{2^{k}}}=\frac{1}{2^{k}-1} .
$$

If $x \in K \backslash\left(C_{k} \cup \alpha^{-n_{k}}\left(B_{k}\right)\right)$, then $x \in K, x \notin C_{k}$ and $\alpha^{n_{k}}(x) \notin B_{k}$. Since $\alpha^{n_{k}}(K) \bigcap K=\varnothing$ and $x \in K$, we have $\alpha^{n_{k}}(x) \in X \backslash K$. Applying the definition of $B_{k}$, we have the following estimate:

$$
\left|\left(f_{k} \circ \alpha^{n_{k}}\right)(x)\right|=\left|f_{k}\left(\alpha^{n_{k}}(x)\right)\right|<\frac{1}{2^{k}}
$$

and so,

$$
\frac{\left|\left(f_{k} \circ \alpha^{n_{k}}\right)(x)\right|}{1-\frac{1}{2^{k}}}<\frac{\frac{1}{2^{k}}}{1-\frac{1}{2^{k}}} .
$$

Moreover, since $x \notin C_{k}$, by the definition of $C_{k}$, one has

$$
1-\left(\prod_{j=0}^{n_{k}-1}\left(w \circ \alpha^{j}\right)(x)\right)\left|\left(f_{k} \circ \alpha^{n_{k}}\right)(x)\right|<\frac{1}{2^{k}}
$$

which says

$$
\left(\prod_{j=0}^{n_{k}-1}\left(w \circ \alpha^{j}\right)(x)\right)^{-1} \leqslant \frac{\left|f_{k} \circ \alpha^{n_{k}}(x)\right|}{1-\frac{1}{2^{k}}} .
$$

Now, (8) can be immediately deduced from (9) and (10).

On the other hand, we are going to show that

$$
\prod_{j=1}^{n_{k}}\left(w \circ \alpha^{-j}\right)(x)<\frac{\frac{1}{2^{k}}}{\left|f_{k}(x)\right|}<\frac{1}{2^{k}-1}, \quad x \in K \backslash\left(D_{k} \cup A_{k}\right) .
$$

With this aim, assume that $x \in K \backslash\left(D_{k} \bigcup A_{k}\right)$. Then $\left|f_{k}(x)\right|>1-\frac{1}{2^{k}}$ by the definition of $A_{k}$. Hence,

$$
\frac{\frac{1}{2^{k}}}{\left|f_{k}(x)\right|}<\frac{\frac{1}{2^{k}}}{1-\frac{1}{2^{k}}}
$$


Also, by the definition of $D_{k}$, we have

$$
\left(\prod_{j=1}^{n_{k}}\left(w \circ \alpha^{-j}\right)(x)\right)\left|f_{k}(x)\right|<\frac{1}{2^{k}}
$$

and so

$$
\prod_{j=1}^{m}\left(w \circ \alpha^{-j}\right)(x)<\frac{\frac{1}{2^{k}}}{\left|f_{k}(x)\right|}
$$

Hence, one can obtain (11) by (12) and (13). Now, put

$$
E_{k}=K \backslash\left(A_{k} \cup \alpha^{-n_{k}}\left(B_{k}\right) \cup C_{k} \cup D_{k}\right)
$$

We claim that

$$
\left\|\chi_{K \backslash E_{k}}\right\|_{\mathcal{F}}<\frac{4}{2^{k}}
$$

Indeed, since

$$
\chi_{K \backslash E_{k}} \leqslant \chi_{A_{k} \cup \alpha^{-n_{k}(B)} \cup C_{k} \cup D_{k}},
$$

we have

$$
\begin{aligned}
\left\|\chi_{K \backslash E_{k}}\right\|_{\mathcal{F}} & \leqslant\left\|\chi_{A_{k}}\right\|_{\mathcal{F}}+\left\|\chi_{\alpha^{-n_{k}\left(B_{k}\right)}}\right\|_{\mathcal{F}}+\left\|\chi_{C_{k}}\right\|_{\mathcal{F}}+\left\|\chi_{D_{k}}\right\|_{\mathcal{F}}= \\
& =\left\|\chi_{A_{k}}\right\|_{\mathcal{F}}+\left\|\chi_{B_{k}}\right\|_{\mathcal{F}}+\left\|\chi_{C_{k}}\right\|_{\mathcal{F}}+\left\|\chi_{D_{k}}\right\|_{\mathcal{F}}<\frac{4}{2^{k}} .
\end{aligned}
$$

Moreover, using (8) and (11), for each $x \in E_{k}$, we have

$$
\left(\prod_{j=0}^{n_{k}-1}\left(w \circ \alpha^{j}\right)(x)\right)^{-1} \leqslant \frac{1}{2^{k}-1} \text { and } \prod_{j=1}^{n_{k}}\left(w \circ \alpha^{-j}\right)(x) \leqslant \frac{1}{2^{k}-1} .
$$

Therefore, condition (ii) follows.

(ii) $\Rightarrow$ (i). Assume that condition (ii) holds. We are going to show that $T_{\alpha, w}$ is topologically transitive. For this, let $U$ and $V$ be non-empty open subsets of $\mathcal{F}$. Since $\mathcal{F}_{b c}$ is dense in $\mathcal{F}$, there are functions $f, g \in \mathcal{F}_{b c}$, such that $f \in U$ and $g \in V$. Put

$$
K:=\operatorname{supp}(f) \bigcup \operatorname{supp}(g) .
$$

Then $K$ is compact. Since $\alpha$ is aperiodic, there is a constant $M>0$ such that for each $n \geqslant M, K \cap \alpha^{ \pm n}(K)=\varnothing$. Besides, for the set $K$, there are a 
sequence of Borel subsets $\left(E_{k}\right)_{k=1}^{\infty}$ of $K$, and a strictly increasing sequence of natural numbers $\left(n_{k}\right)_{k=1}^{\infty}$ satisfying condition (ii). Here we may assume $n_{k}>M$ for each $k \in \mathbb{N}$. Thus,

$$
\begin{aligned}
& \left\|T_{\alpha, w}^{n_{k}}\left(f \chi_{E_{k}}\right)\right\|_{\mathcal{F}}=\left\|\left(\prod_{j=0}^{n_{k}-1}\left(w \circ \alpha^{j}\right)\right) \cdot\left(f \chi_{E_{k}}\right) \circ \alpha^{n_{k}}\right\|_{\mathcal{F}}= \\
& =\left\|\left(\prod_{j=1}^{n_{k}}\left(w \circ \alpha^{-j}\right)\right) \cdot\left(f \chi_{E_{k}}\right)\right\|_{\mathcal{F}} \leqslant\|f\|_{\mathcal{F}} \cdot \sup _{x \in E_{k}} \prod_{j=1}^{n_{k}}\left(w \circ \alpha^{-j}\right)(x)
\end{aligned}
$$

for each $k \in \mathbb{N}$. Hence, by condition (ii),

$$
\lim _{k \rightarrow \infty}\left\|T_{\alpha, w}^{n_{k}}\left(f \chi_{E_{k}}\right)\right\|_{\mathcal{F}}=0 .
$$

Similarly,

$$
\lim _{k \rightarrow \infty}\left\|S_{\alpha, w}^{n_{k}}\left(g \chi_{E_{k}}\right)\right\|_{\mathcal{F}}=0
$$

For each $k \in \mathbb{N}$, let

$$
v_{k}:=f \chi_{E_{k}}+S_{\alpha, w}^{n_{k}}\left(g \chi_{E_{k}}\right) .
$$

Then, $v_{k} \in \mathcal{F}$ and, for each $k \in \mathbb{N}$, we have

$$
\begin{aligned}
\left\|v_{k}-f\right\|_{\mathcal{F}} & \leqslant\left\|f-f \chi_{E_{k}}\right\|_{\mathcal{F}}+\left\|S_{\alpha, w}^{n_{k}}\left(g \chi_{E_{k}}\right)\right\|_{\mathcal{F}}= \\
& =\left\|f \cdot \chi_{K \backslash E_{k}}\right\|_{\mathcal{F}}+\left\|S_{\alpha, w}^{n_{k}}\left(g \chi_{E_{k}}\right)\right\|_{\mathcal{F}} \leqslant \\
& \leqslant\|f\|_{\sup }\left\|\chi_{K \backslash E_{k}}\right\|_{\mathcal{F}}+\left\|S_{\alpha, w}^{n_{k}}\left(g \chi_{E_{k}}\right)\right\|_{\mathcal{F}}
\end{aligned}
$$

So, $\lim _{k \rightarrow \infty} v_{k}=f$ in $\mathcal{F}$. Also, by (14), we have $\lim _{k \rightarrow \infty} T_{\alpha, w}^{n_{k}} v_{k}=g$ in $\mathcal{F}$, which is implied by the inequalities $|g|\left(1-\chi_{E_{k}}\right) \leqslant|g| \chi_{K \backslash E_{k}}$ and

$$
\begin{aligned}
\left\|T_{\alpha, w}^{n_{k}} v_{k}-g\right\|_{\mathcal{F}}=\| T_{\alpha, w}^{n_{k}}\left(f \chi_{E_{k}}\right)+ & g \chi_{E_{k}}-g \|_{\mathcal{F}} \leqslant \\
& \leqslant\left\|T_{\alpha, w}^{n_{k}}\left(f \chi_{E_{k}}\right)\right\|_{\mathcal{F}}+\|g\|_{\text {sup }}\left\|\chi_{K \backslash E_{k}}\right\|_{\mathcal{F}} .
\end{aligned}
$$

Therefore, $U \cap T^{-n_{k}}(V) \neq \varnothing$. This implies that $T_{\alpha, w}$ is topologically transitive, and the proof is complete.

In the following, we give some sufficient and necessary conditions for a weighted translation to be chaotic. First we recall the definition of chaos. Definition 7. Let $\mathcal{X}$ be a Banach space, and $T$ be a bounded linear operator on $\mathcal{X}$. A vector $x \in \mathcal{X}$ is called a periodic element of $T$ if there 
exists a constant $N \in \mathbb{N}$, such that $T^{N} x=x$. The set of all periodic elements of $T$ is denoted by $\mathcal{P}(T)$. An operator $T$ is called chaotic if it is topologically transitive and $\mathcal{P}(T)$ is dense in $\mathcal{X}$.

Theorem 2. Let $X$ be a topological space, $\alpha$ be a Borel measurable bijection from $X$ onto $X$, such that $\alpha^{-1}$ is also Borel measurable, and let $w$ be a weight on $X$. Let $\mathcal{F}$ be a Banach function space on $X$ satisfying condition $\Omega_{\alpha}$. Assume that for each compact subset $E \subseteq X$, there exists a sequence of Borel subsets $\left(E_{k}\right)_{k=1}^{\infty}$ of $K$, such that $\lim _{k \rightarrow \infty}\left\|\chi_{K \backslash E_{k}}\right\|_{\mathcal{F}}=0$ and

$$
\begin{aligned}
\lim _{k \longrightarrow \infty}\left(\sum_{l=1}^{\infty}\left\|\left(\prod_{j=1}^{l n_{k}} w \circ \alpha^{-j}\right) \cdot \chi_{E_{k}}\right\|_{\mathcal{F}}+\right. & \\
& \left.+\sum_{l=1}^{\infty}\left\|\left(\prod_{j=0}^{l n_{k}-1} w \circ \alpha^{j}\right)^{-1} \cdot \chi_{E_{k}}\right\|_{\mathcal{F}}\right)=0
\end{aligned}
$$

for some strictly increasing sequence $\left(n_{k}\right)_{k=1}^{\infty} \subseteq \mathbb{N}$. Then $T_{\alpha, w}$ is chaotic on $\mathcal{F}$.

Proof. First we show that $T_{\alpha, w}$ is topologically transitive. Let $U$ and $V$ be non-empty open subsets of $\mathcal{F}$. We prove that there exists a number $n \in \mathbb{N}$, such that $T_{\alpha, w}^{n}(U) \cap V \neq \varnothing$. Since $\mathcal{F}_{b c}$ is dense in $\mathcal{F}$, there are functions $f, g$, such that $f \in U \cap \mathcal{F}_{b c}$ and $g \in V \cap \mathcal{F}_{b c}$. Put $K:=\operatorname{supp}(f) \cup \operatorname{supp}(g)$. So, $K$ is compact. Then we can pick an increasing sequence $\left(n_{k}\right) \subseteq \mathbb{N}$ and a sequence of subsets $\left(E_{k}\right)$ of $K$ satisfying the assumptions of the statement. Due to the inequality

$$
\begin{gathered}
\left\|T_{\alpha, w}^{n_{k}}\left(f \chi_{E_{k}}\right)\right\|_{\mathcal{F}}=\left\|\left(\prod_{j=0}^{n_{k}-1} w \circ \alpha^{j}\right) \cdot\left(f \chi_{E_{k}}\right) \circ \alpha^{n_{k}}\right\|_{\mathcal{F}}= \\
=\left\|\left(\prod_{j=1}^{n_{k}} w \circ \alpha^{-j}\right) \cdot\left(f \chi_{E_{k}}\right)\right\|_{\mathcal{F}} \leqslant \\
\leqslant\|f\|_{\sup }\left\|\left(\prod_{j=1}^{n_{k}} w \circ \alpha^{-j}\right) \cdot \chi_{E_{k}}\right\|_{\mathcal{F}}
\end{gathered}
$$

one has $\lim _{k \rightarrow \infty} T_{\alpha, w}^{n_{k}}\left(f \chi_{E_{k}}\right)=0$ in $\mathcal{F}$. Similar to the above argument, we can see that $\lim _{k \rightarrow \infty} S_{\alpha, w}^{n_{k}}\left(g \chi_{E_{k}}\right)=0$ in $\mathcal{F}$. (Note that in this theorem, it is 
not necessary to assume that $\frac{1}{w}$ is bounded. This is because here $f$ and $g$ are compactly supported.) Therefore, together with $\lim _{k \rightarrow \infty}\left\|\chi_{K \backslash E_{k}}\right\|_{\mathcal{F}}=0$, we have

$\lim _{k \rightarrow \infty}\left(S_{\alpha, w}^{n_{k}}\left(g \chi_{E_{k}}\right)+f \chi_{E_{k}}\right)=f \quad$ and $\quad \lim _{k \rightarrow \infty} T_{\alpha, w}^{n_{k}}\left(S_{\alpha, w}^{n_{k}}\left(g \chi_{E_{k}}\right)+f \chi_{E_{k}}\right)=g$

This implies that $T_{\alpha, w}^{n_{k}}(U) \cap V \neq \varnothing$ for some $k \in \mathbb{N}$, which tells us that $T_{\alpha, w}$ is topologically transitive.

Next, we prove that $\mathcal{P}\left(T_{\alpha, w}\right)$ is dense in $\mathcal{F}$. Recall that $f \in U \cap \mathcal{F}_{b c}$. For each $k \in \mathbb{N}$, let

$$
v_{k}:=f \chi_{E_{k}}+\sum_{l=1}^{\infty} T_{\alpha, w}^{l n_{k}}\left(f \chi_{E_{k}}\right)+\sum_{l=1}^{\infty} S_{\alpha, w}^{l n_{k}}\left(f \chi_{E_{k}}\right) .
$$

Note that the series $\sum_{l=1}^{\infty} T_{\alpha, w}^{l n_{k}}\left(f \chi_{E_{k}}\right)$ is convergent in $\mathcal{F}$ because it is absolutely convergent. Indeed,

$$
\sum_{l=1}^{\infty}\left\|T_{\alpha, w}^{l n_{k}}\left(f \chi_{E_{k}}\right)\right\|_{\mathcal{F}} \leqslant\|f\|_{\text {sup }} \sum_{l=1}^{\infty}\left\|\left(\prod_{j=0}^{l n_{k}} w \circ \alpha^{-j}\right) \cdot \chi_{E_{k}}\right\|_{\mathcal{F}}<\infty .
$$

Similarly, $\sum_{l=1}^{\infty} S_{\alpha, w}^{l n_{k}}\left(f \chi_{E_{k}}\right)$ is convergent in $\mathcal{F}$, too. Using $\lim _{k \rightarrow \infty}\left\|\chi_{K \backslash E_{k}}\right\|_{\mathcal{F}}=0$, we have $\lim _{k \rightarrow \infty} v_{k}=f$ in $\mathcal{F}$. In addition, a simple calculation gives $T_{\alpha, w}^{n_{k}} v_{k}=v_{k}$. This implies that $U \cap P\left(T_{\alpha, w}\right) \neq \varnothing$. Therefore, $\mathcal{P}\left(T_{\alpha, w}\right)$ is dense in $\mathcal{F}$.

Example 4. Let $1 \leqslant q \leqslant p<\infty$. Let $f \in L_{\mathrm{loc}}^{q}\left(\mathbb{R}^{n}\right)$. Then its Morrey norm is defined by

$$
\|f\|_{\mathcal{M}_{q}^{p}}:=\sup _{(x, r) \in \mathbb{R}^{n} \times(0, \infty)}|B(x, r)|^{\frac{1}{p}-\frac{1}{q}}\left(\int_{B(x, r)}|f(y)|^{q} \mathrm{~d} y\right)^{\frac{1}{q}},
$$

where $B(x, r)$ is the open ball centered by $x$ with radius $r$. In this case, the set of all $L_{\text {loc }}^{q}\left(\mathbb{R}^{n}\right)$-functions $f$ with $\|f\|_{\mathcal{M}_{q}^{p}}<\infty$ is denoted by $\mathcal{M}_{q}^{p}\left(\mathbb{R}^{n}\right)$, and $\left(\mathcal{M}_{q}^{p}\left(\mathbb{R}^{n}\right),\|\cdot\|_{\mathcal{M}_{q}^{p}}\right)$ is called a Morrey space. Morrey spaces are generalizations of the usual Lebesgue spaces. In fact, for each $1 \leqslant p<\infty$, we have $\mathcal{M}_{p}^{p}\left(\mathbb{R}^{n}\right)=L^{p}\left(\mathbb{R}^{n}\right)$. Morrey spaces were introduced by J. Peetre in [13], which was originally motivated by [12]. For more details and references, see [15-17]. For each $1 \leqslant q<p<\infty$, the Morrey space 
$\mathcal{M}_{q}^{p}\left(\mathbb{R}^{n}\right)$ is not separable. However, if we consider the $\mathcal{M}_{q}^{p}\left(\mathbb{R}^{n}\right)$-closure of $L_{\mathrm{c}}^{\infty}\left(\mathbb{R}^{n}\right) \cap \mathcal{M}_{q}^{p}\left(\mathbb{R}^{n}\right)$, denoted by $\widetilde{\mathcal{M}}_{q}^{p}\left(\mathbb{R}^{n}\right)$, then the Banach space $\widetilde{\mathcal{M}}_{q}^{p}\left(\mathbb{R}^{n}\right)$ is separable and infinite-dimensional, where $L_{\mathrm{c}}^{\infty}\left(\mathbb{R}^{n}\right)$ is the set of all functions in $L^{\infty}\left(\mathbb{R}^{n}\right)$ with compact support. Also, for each $1 \leqslant q<p<\infty$, the set $\widetilde{\mathcal{M}}_{q}^{p}\left(\mathbb{R}^{n}\right) \backslash L^{p}\left(\mathbb{R}^{n}\right)$ is spaceable and large enough (see [5, Theorem 2.2]). Note that a subset $S$ of a topological vector space $X$ is called spaceable if $S \cup\{0\}$ contains a closed infinite-dimensional subspace of $X$.

As we mentioned in Example 1, for each non-zero element $a \in \mathbb{R}^{n}$, the function $\alpha_{a}$ defined by $\alpha_{a}(x):=x-a$ for all $x \in \mathbb{R}^{n}$ is an aperiodic function. Therefore, by the above facts, the Banach space $\widetilde{\mathcal{M}}_{q}^{p}\left(\mathbb{R}^{n}\right)$ satisfies the condition $\Omega_{\alpha_{a}}$, because the Lebesgue measure on $\mathbb{R}^{n}$ is additive-invariant.

Applying Theorem 2 and Example 4, we can conclude the following result for Morrey spaces.

Corollary 1. Let $w$ be a weight on $\mathbb{R}^{n}$, and let $a \in \mathbb{R}^{n}$ be a non-zero element. Assume that for each compact subset $K \subseteq \mathbb{R}^{n}$, there exists a sequence of Borel subsets $\left(E_{k}\right)_{k=1}^{\infty}$ of $K$, such that $\lim _{k \rightarrow \infty}\left\|\chi_{K \backslash E_{k}}\right\|_{\mathcal{M}_{q}^{p}}=0$ and

$$
\begin{aligned}
\lim _{k \longrightarrow \infty}\left(\sum_{l=1}^{\infty}\left\|\left(\prod_{j=1}^{l n_{k}} w(\cdot-j a)\right) \cdot \chi_{E_{k}}\right\|_{\mathcal{M}_{q}^{p}}+\right. \\
\left.+\sum_{l=1}^{\infty}\left\|\left(\prod_{j=0}^{l n_{k}-1} w(\cdot+j a)\right)^{-1} \cdot \chi_{E_{k}}\right\|_{\mathcal{M}_{q}^{p}}\right)=0
\end{aligned}
$$

for some strictly increasing sequence $\left(n_{k}\right)_{k=1}^{\infty} \subseteq \mathbb{N}$. Then $T_{\alpha, w}$ is chaotic on $\widetilde{\mathcal{M}}_{q}^{p}\left(\mathbb{R}^{n}\right)$.

We end this paper by giving a necessary condition for chaos under one more assumption. For each $f \in \mathcal{F}$, let $\sigma(f):=\{x \in X: f(x) \neq 0\}$. In Theorem 3, we assume that there exists a constant $p>0$, such that $\|f+g\|_{\mathcal{F}}^{p}=\|f\|_{\mathcal{F}}^{p}+\|g\|_{\mathcal{F}}^{p}$ whenever $f, g \in \mathcal{F}$ and $\sigma(f) \cap \sigma(g)=\varnothing$. In fact, this property appears in many familiar Banach spaces.

Theorem 3. Let $X$ be a topological space, $\alpha$ be an aperiodic function from $X$ onto $X$, and let $w$ be a weight on $X$. Let $\mathcal{F}$ be a Banach function space on $X$ satisfying condition $\Omega_{\alpha}$ and the additional condition above. Assume that $T_{\alpha, w}$ is chaotic on $\mathcal{F}$. Then, for each compact subset $K \subseteq X$, there exists a sequence of Borel subsets $\left(E_{k}\right)_{k=1}^{\infty}$ of $K$, such that 
$\lim _{k \rightarrow \infty}\left\|\chi_{K \backslash E_{k}}\right\|_{\mathcal{F}}=0$ and

$$
\begin{aligned}
\lim _{k \rightarrow \infty}\left(\sum_{l=1}^{\infty}\left\|\left(\prod_{j=1}^{l n_{k}} w \circ \alpha^{-j}\right) \cdot \chi_{E_{k}}\right\|_{\mathcal{F}}^{p}+\right. & \\
& \left.+\sum_{l=1}^{\infty}\left\|\left(\prod_{j=0}^{l n_{k}-1} w \circ \alpha^{j}\right)^{-1} \cdot \chi_{E_{k}}\right\|_{\mathcal{F}}^{p}\right)=0
\end{aligned}
$$

for some strictly increasing sequence $\left(n_{k}\right)_{k=1}^{\infty} \subseteq \mathbb{N}$.

Proof. Suppose that $T_{\alpha, w}$ is chaotic and $K \subseteq X$ is compact. Then, by the condition $\Omega_{\alpha}$, we have $\chi_{K} \in \mathcal{F}$. Since $\alpha$ is an aperiodic function, there exists a constant $M>0$, such that for each $n \geqslant M$,

$$
K \cap \alpha^{ \pm n}(K)=\varnothing .
$$

For each $k \in \mathbb{N}$, by the density of periodic elements of $T_{\alpha, w}$, there exists $f_{k} \in \mathcal{P}\left(T_{\alpha, w}\right)$, such that $T_{\alpha, w}^{n_{k}} f_{k}=f_{k}=S_{\alpha, w}^{n_{k}} f_{k}$ and

$$
\left\|f_{k}-\chi_{K}\right\|_{\mathcal{F}}<\frac{1}{4^{k}}
$$

where we may assume that $n_{1}>M$, and $\left(n_{k}\right)_{k=1}^{\infty}$ is strictly increasing. Put $A_{k}:=\left\{x \in K:\left|f_{k}(x)-1\right| \geqslant \frac{1}{2^{k}}\right\}$. Then, as in the proof of Theorem 1, we have

$$
\left|f_{k}(x)\right|>1-\frac{1}{2^{k}} \quad \text { on } \quad K \backslash A_{k}, \quad \text { and } \quad\left\|\chi_{A_{k}}\right\|_{\mathcal{F}}<\frac{1}{2^{k}} .
$$

Let $E_{k}=K \backslash A_{k}$. Then

$$
\left\|\chi_{K \backslash E_{k}}\right\|_{\mathcal{F}}=\left\|\chi_{A_{k}}\right\|_{\mathcal{F}}<\frac{1}{2^{k}} .
$$

On the other hand, by the fact $K \cap \alpha^{ \pm n_{k}}(K)=\varnothing$, we observe

$$
\begin{aligned}
& \left|\chi_{\bigcup_{l=1}^{\infty}\left(\alpha^{-l n_{k}}(K) \cup \alpha^{l n_{k}}(K)\right)} f_{k}\right| \\
& =\left|\chi_{\bigcup_{l=1}^{\infty}\left(\alpha^{-l n_{k}}(K)\right.} \cup \alpha^{\left.l n_{k}(K)\right)} f_{k}-\chi_{\bigcup_{l=1}^{\infty}\left(\alpha^{-l n_{k}}(K) \cup \alpha^{l n_{k}}(K)\right)} \chi_{K}\right|= \\
& =\chi_{\bigcup_{l=1}^{\infty}\left(\alpha^{-l n_{k}}(K) \cup \alpha^{\left.l n_{k}(K)\right)}\right.}\left|f_{k}-\chi_{K}\right| \leqslant\left|f_{k}-\chi_{K}\right| .
\end{aligned}
$$

It follows that

$$
\left\|\chi_{\bigcup_{l=1}^{\infty}\left(\alpha^{-l n_{k}}(K) \cup \alpha^{\left.l n_{k}(K)\right)}\right.} f_{k}\right\|_{\mathcal{F}} \leqslant\left\|f_{k}-\chi_{K}\right\|_{\mathcal{F}}
$$


Hence, by $\alpha$-invariance, and the disjointness of $\alpha^{r n_{k}}(K)$ and $\alpha^{s n_{k}}(K)$ for $r \neq s$, we get

$$
\begin{gathered}
\frac{1}{4^{k p}}>\left\|\chi_{\cup_{l=1}^{\infty}\left(\alpha^{-l n_{k}}(K) \cup \alpha^{\left.l n_{k}(K)\right)}\right.} f_{k}\right\|_{\mathcal{F}}^{p}=\left\|\sum_{l=1}^{\infty} \chi_{\alpha^{-l n_{k}(K)}} f_{k}+\sum_{l=1}^{\infty} \chi_{\alpha^{l n_{k}(K)}} f_{k}\right\|_{\mathcal{F}}^{p}= \\
=\sum_{l=1}^{\infty}\left\|\chi_{\alpha^{-l n_{k}(K)}} f_{k}\right\|_{\mathcal{F}}^{p}+\sum_{l=1}^{\infty}\left\|\chi_{\alpha^{l n_{k}(K)}} f_{k}\right\|_{\mathcal{F}}^{p}= \\
=\sum_{l=1}^{\infty}\left\|\chi_{K}\left(f_{k} \circ \alpha^{-l n_{k}}\right)\right\|_{\mathcal{F}}^{p}+\sum_{l=1}^{\infty}\left\|\chi_{K}\left(f_{k} \circ \alpha^{l n_{k}}\right)\right\|_{\mathcal{F}}^{p}= \\
=\sum_{l=1}^{\infty}\left\|\chi_{K}\left(\left(T_{\alpha, w}^{l n_{k}} f_{k}\right) \circ \alpha^{-l n_{k}}\right)\right\|_{\mathcal{F}}^{p}+\sum_{l=1}^{\infty}\left\|\chi_{K}\left(\left(S_{\alpha, w}^{l n_{k}} f_{k}\right) \circ \alpha^{l n_{k}}\right)\right\|_{\mathcal{F}}^{p}= \\
=\sum_{l=1}^{\infty}\left\|\chi_{K}\left(\prod_{j=0}^{l n_{k}-1} w \circ \alpha^{j} \circ \alpha^{-l n_{k}}\right) \cdot\left(f_{k} \circ \alpha^{l n_{k}} \circ \alpha^{-l n_{k}}\right)\right\|_{\mathcal{F}}^{p}+ \\
+\sum_{l=1}^{\infty}\left\|\chi_{K}\left(\prod_{j=1}^{l n_{k}} w \circ \alpha^{-j} \circ \alpha^{l n_{k}}\right)^{-1} \cdot\left(f_{k} \circ \alpha^{-l n_{k}} \circ \alpha^{l n_{k}}\right)\right\|_{\mathcal{F}}^{p}
\end{gathered}
$$

This implies that

$$
\begin{aligned}
& \frac{1}{4^{k p}}>\sum_{l=1}^{\infty}\left\|\left(\prod_{j=1}^{l n_{k}} w \circ \alpha^{-j}\right) \cdot f_{k} \chi_{E_{k}}\right\|_{\mathcal{F}}^{p}+\sum_{l=1}^{\infty}\left\|\left(\prod_{j=0}^{l n_{k}-1} w \circ \alpha^{j}\right)^{-1} \cdot f_{k} \chi_{E_{k}}\right\|_{\mathcal{F}}^{p}> \\
& >\left(1-\frac{1}{2^{k}}\right)\left(\sum_{l=1}^{\infty}\left\|\left(\prod_{j=1}^{l n_{k}} w \circ \alpha^{-j}\right) \cdot \chi_{E_{k}}\right\|_{\mathcal{F}}^{p}+\sum_{l=1}^{\infty}\left\|\left(\prod_{j=0}^{l n_{k}-1} w \circ \alpha^{j}\right)^{-1} \cdot \chi_{E_{k}}\right\|_{\mathcal{F}}^{p}\right) .
\end{aligned}
$$

Therefore, the condition given in the conclusion follows.

Acknowledgment. We would like to thank the referee for carefully reading our manuscript and for his/her helpful comments.

\section{References}

[1] Ansari S. I. Existence of hypercyclic operators on topological vector spaces. J. Funct. Anal., 1997, 148, pp. 384-390.

DOI: https://doi.org/10.1006/jfan.1996.3093 
[2] Bayart F., Matheron É. Dynamics of Linear Operators. Cambridge Tracts in Math., 179, Cambridge University Press, Cambridge, 2009.

[3] Bennett C., Sharpley R. Interpolation of Operators. Academic Press Inc., Boston, 1988.

[4] Bernal-González L. On hypercyclic operators on Banach spaces. Proc. Amer. Math. Soc., 1999, 127, pp. 1003-1010.

DOI: https://doi.org/10.1090/S0002-9939-99-04657-2

[5] Bernal-González L., Cabrera M. O. Spaceability of strict order integrability. J. Math. Anal. Appl., 2012, 385, pp. 303-309.

DOI: https://doi.org/10.1016/j.jmaa.2011.06.043

[6] Chen C-C., Chu C-H. Hypercyclicity of weighted convolution operators on homogeneous spaces. Proc. Amer. Math. Soc., 2009, 137, pp. 2709-2718.

DOI: https://doi.org/10.1090/S0002-9939-09-09889-X

[7] Chen C-C., Chu C-H. Hypercyclic weighted translations on groups. Proc. Amer. Math. Soc., 2011, 139, pp. 2839-2846.

DOI: https://doi.org/10.1090/S0002-9939-2011-10718-4

[8] Chen C-C., Chen K-Y., Öztop S., Tabatabaie S. M. Chaotic translations on weighted Orlicz spaces. Ann. Polon. Math., 2019, 122, 129-142.

DOI: https://doi.org/10.4064/ap180910-21-1

[9] Chen C-C., Öztop S., Tabatabaie S. M. Disjoint dynamics on weighted Orlicz spaces. Complex Anal. Oper. Theory, 2020, 14, Paper No. 72, 18 pp. DOI: https://doi.org/10.1007/s11785-020-01034-x

[10] Grosse-Erdmann K.-G., Peris A. Linear Chaos. Universitext, Springer, 2011.

[11] Hewitt E., Ross K. A. Abstract Harmonic Analysis. Springer-Verlag, Heidelberg, 1979.

[12] Morrey C. B. On the solutions of quasi linear elliptic partial differential equations. Trans. Amer. Math. Soc., 1938, 43, pp. 126 - 166.

DOI: https://doi.org/10.1090/S0002-9947-1938-1501936-8

[13] Peetre J. On the theory of $\mathcal{L}_{p, \lambda}$. J. Func. Anal., 1969, 4, pp. $71-87$.

DOI: https://doi.org/10.1016/0022-1236(69)90022-6

[14] Salas H. Hypercyclic weighted shifts. Trans. Amer. Math. Soc., 1995, 347, pp. 993-1004.

DOI: https://doi.org/10.1090/S0002-9947-1995-1249890-6

[15] Sawano Y. A non-dense subspace in $\mathcal{M}_{q}^{p}$ with $1<q<p<\infty$. Trans. A. Razmadze Math. Inst., 2017, 171, pp. $379-380$.

DOI: https://doi.org/10.1016/j.trmi.2017.05.001 
[16] Sawano Y., Sugano S., Tanaka H. Generalized fractional integral operators and fractional maximal operators in the framework of Morrey spaces. Trans. Amer. Math. Soc., 2011, 363, pp. 6481 - 6503.

DOI: https://doi.org/10.1090/S0002-9947-2011-05294-3

[17] Triebel H. Hybrid Function Spaces, Heat and Navier-Stokes Equations. Tracts in Mathematics 24, 2015, European Mathematical Society.

Received July 16, 2020.

In revised form, November 02, 2020.

Accepted November 03, 2020.

Published online November 15, 2020.

\section{C-C. Chen}

Department of Mathematics Education, National Taichung University of Education, No.140, Minsheng Rd., West Dist., Taichung 40306, Taiwan E-mail: chungchuan@mail.ntcu.edu.tw

S. M. Tabatabaie

Department of Mathematics, Faculty of Science, University of Qom, Alghadir Blvd, Qom 3716146611, Iran

E-mail:sm.tabatabaie@qom.ac.ir 